\title{
Percepciones de los estudiantes sobre el curso de Bioética para la especialidad de Biología y de Química en el IPC-UPEL
}

\author{
Students' perceptions on the Bioethics course for the specialty of Biology \\ and Chemistry at the IPC-UPEL
}

Recibido: 22 de julio de 2019 / Aprobado: 21 de mayo de 2020

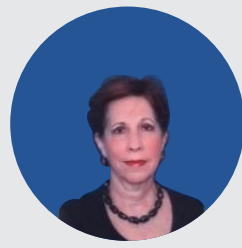

MARLENE OCHOA DE TOLEDO UNIVERSIDAD PEDAGÓGICA EXPERIMENTAL LIBERTADOR, CARACAS marlene8atoledo@gmail.com

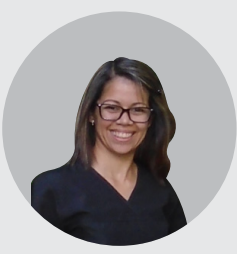

GELVIS ALVARADO

UNIVERSIDAD PEDAGÓGICA EXPERIMENTAL LIBERTADOR, CARACAS gelvisalexandra@gmail.com

\section{Resumen}

En este trabajo se presenta el relato sobre la experiencia de implementación de un curso de Bioética para estudiantes del Instituto Pedagógico de Caracas, en donde se aplicaron estrategias enmarcadas en la reflexión crítica. El objetivo fundamental fue analizar las percepciones de los educandos para establecer el logro de los objetivos del curso, el rol del docente y determinar la aceptación de las estrategias manejadas. Para el análisis, el trabajo se fundamentó en el modelo de Müller y Szczureck el cual consta de las siguientes fases: 1. Diagnóstico de necesidades 2. Diseño de la propuesta 3. Validación de la propuesta. 4. Evaluación de los resultados de la implementación de la propuesta. La presente investigación se centra en las etapas 3 y 4 , aunque describe brevemente las estrategias metodológicas que se utilizaron en el diseño, elemento correspondiente de la fase 2. La tercera etapa de validación se realizó a partir de una aplicación piloto de un curso de Bioética para futuros docentes de Biología en el semestre 2009-I. Este estudio utiliza una metodología cuasi experimental cualitativa con estudio de campo y se enmarca en el paradigma interpretativo porque se pretende recabar la información de los estudiantes para su posterior análisis e interpretación de la realidad. Los resultados obtenidos fueron la base para la cuarta etapa de análisis de resultados de la implementación que muestran una total aceptación del curso evidenciada a través de sus percepciones; igualmente, los estudiantes (futuros docentes de Biología y de Química) señalan que el aprendizaje logrado se debe a las estrategias utilizadas para los análisis bioéticos, la motivación generada por el curso y la participación en todas las clases, lo que les posibilitó cambios conceptuales señalados a través de reflexiones críticas. Estos aspectos llevan a concluir que posiblemente las estrategias utilizadas pueden promover cambios en las concepciones a partir de un aprendizaje crítico.

Palabras clave: aprendizaje crítico; bioética; estrategias docentes.

\footnotetext{
a. Profesor Titular Jubilado del Instituto Pedagógico de Caracas, Instituto Pedagógico de Caracas, Universidad Pedagógica Experimental Libertador, Caracas. Para contactar a la autora: marlene8atoledo@gmail.com

b. Supervisora del laboratorio de Microscopía del Departamento de Biología y Química. Instituto Pedagógico de Caracas, Universidad Pedagógica Experimental Libertador, Caracas. Para contactar a la autora: gelvisalexandra@gmail.com
} 


\section{Abstract}

This paper presents the account of the experience of implementing a bioetic course for students of the Instituto Pedagógico deCaracas, where strategies framed in critical reflection were applied. The fundamental objective was to analyze the perceptions of the students to establish the achievement of the objectives of the course, the role of the teacher and determine the acceptance of the strategies used. For the analysis, the work was based on the Müller and Szczureck model which consists of the following phases: 1. Diagnosis of needs 2. Design of the proposal 3. Validation of the proposal. 4. Evaluation of the results of the implementation of the proposal. This research focuses on stages 3 and 4, although it briefly describes the methodological strategies used in the design, a corresponding element of phase 2 . The third stage of validation was carried out from a pilot application of a Bioethics course for future Biology teachers in the 2009-I semester. This study uses a quasi-experimental qualitative methodology with a field study and is framed in the interpretive paradigm because it is intended to collect information from students for their subsequent analysis and interpretation of reality. The results obtained were the basis for the fourth stage of analysis of implementation results that show total acceptance of the course evidenced through their perceptions; likewise, the students (future teachers of Biology and Chemistry) point out that the learning achieved is due to the strategies used for bioethical analyzes, the motivation generated by the course and participation in all classes, which enabled them to make conceptual changes through critical reflections. These aspects lead to the conclusion that possibly the strategies used can promote changes in conceptions based on critical learning.

Keywords: critical learning; bioethics; teaching strategies.

\section{Introducción}

Si bien el término Bioética parece nuevo, en realidad es tan antiguo como el hombre (Molina, 2011), ya que representa un espacio para la reflexión articulada en una escala de valores y principios morales para la toma de decisiones responsables acerca de problemas relacionados con la vida biológica, el ambiente y los desarrollos de la ciencia (Bermúdez, 2006; Cerchiaro, 2011). A pesar de que el término se propuso en 1927, se popularizó con Potter (considerado el padre de la bioética) quien lo utiliza en 1970 para identificar una nueva disciplina que representa el puente entre la ética clásica y las ciencias de la vida (Molina, 2011; Wilches Flórez, 2011). Por algunos años, pareció que la bioética se limitaba al área de la salud, pero en los últimos tiempos se retoma a la bioética en un concepto más amplio, tal como la definen Pessina (Catedrático de Bioética en la Universidad del Sacro Cuore de Milán) como "conciencia crítica de la civilización tecnológica", y, Postigo, quien considera a la bioética como el estudio sistemático e interdisciplinario de las implicaciones éticas de las acciones humanas sobre todo tipo de vida para determinar una posible solución para el hombre y las generaciones futuras (Postigo, 2015).

Aunque la bioética abarca varios ámbitos, es importante recordar su raíz filosófica, que a su vez tiene dos vertientes: la antropológica, mediante la cual se define al hombre y la ética. (Postigo, 2015). La vertiente antropológica establece la perspectiva de la bioética materialista, que va acorde al utilitarismo de Stuart Mill (Sánchez-Migallón, 2012) o, personalista, como la plantea Sgreccia (2013) que tiene como centro el bien de la persona (Postigo, 2015). Estas perspectivas han conllevado a la derivación de diversas corrientes, entre ellas el Principialismo, que se fundamenta en los cuatro principios de Childress y Beauchamp (Royes y Qui, 2019), y no considera una teoría antropológica; esta es 
la que maneja la cultura angloamericana. Otra corriente es la del Personalismo con fundamentación antropológica, que afirma que el hombre es la unión de cuerpo y alma y que la vida comienza con la fecundación (Postigo, 2015). Finalmente, corrientes como el Utilitarismo y el Funcionalismo materialista consideran que lo bueno es lo que favorece al mayor número de personas (Sánchez-Migallón, 2012); ambas son manejadas por la cultura europea.

Lo importante de estas corrientes es el pluralismo que implica la incorporación de la bioética como tema en la investigación; por esto es necesario el logro de acuerdos a nivel de la comunidad científica a fin de asegurar el respeto y la promoción de los derechos humanos, los cuales representan el límite y el criterio orientador para tales acuerdos (Casado, 2011). Al respecto, se han planteado códigos compuestos por declaraciones que establecen pautas de la bioética en sus diversas aplicaciones.

Una de esas aplicaciones sería en la educación, ya que si es parte del desarrollo humano, es importante incluir la educación en bioética para formar ciudadanos capaces de tomar decisiones frente a los avances científicos y tecnológicos, lo que implica diseñar propuestas de aula para propiciar esa formación (Orozco, 2016). Por eso, se consideró la implementación de un curso de Bioética en el Instituto Pedagógico de Caracas (IPC). El modelo para el diseño e implantación del curso fue el propuesto por Müller de González y Szczurek en 1989, quienes presentan un modelo en la aplicación del Desarrollo Instruccional (citado por Castro y Guzmán de Castro, 2001). En la figura 1 pueden observarse los pasos del modelo:

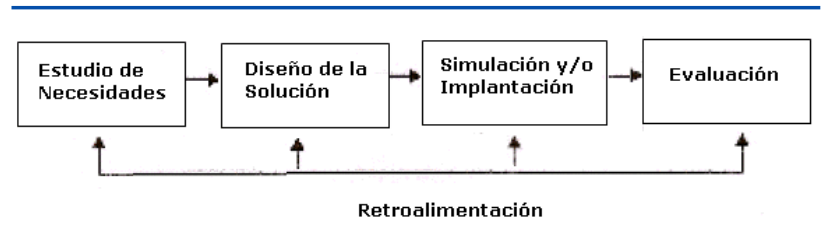

Figura 1. Modelo de Desarrollo Tecnológico. Extraído del material del curso "Desarrollo Instruccional" propuesto por Müller de González, G. y Szczurek, M. (1989) (citado en Castro y Guzmán de Castro, 2001).
Siguiendo este modelo, en la publicación de Alvarado, Partida y Ochoa de Toledo (2017) se describen los pasos 1 y 2. En cuanto al 1, se señalan los elementos que se consideraron para establecer la necesidad de un curso de bioética: primero, estaba la experiencia docente en el curso de Ciencia, Tecnología y Sociedad (CTS), cátedra en la que muchas veces se presentaban discusiones que implicaban valores; sin embargo, estas solo se centraban en la valoración personal, lo que creó la necesidad de brindar herramientas a los futuros docentes en cuanto al análisis de dilemas bioéticos, ya que faltaban elementos que contribuyeran a la objetividad de las argumentaciones.

Segundo, el artículo 23 de la Declaración Universal sobre Bioética y Derechos Humanos tiene como principal objetivo la promoción de los principios de la declaración mediante programas de educación a fin de capacitar a los ciudadanos en las implicaciones bioéticas de la ciencia y de la tecnología (Casado, 2011); al respecto, las políticas gubernamentales venezolanas en la Ley Orgánica de Educación (2009), en el artículo 33, señala a "la bioética como principio rector de la educación universitaria" y, el Plan Socialista de la Nación (2007-2013), apoya esta inclusión en la educación venezolana y va más allá cuando propone la transversalización de la Bioética en la educación; más aún, desde 2011 se cuenta con el Código de Ética para la Vida del Ministerio de Ciencia y Tecnología de la República Bolivariana de Venezuela (2011).

Establecida la necesidad, el siguiente reto fue diseñar un curso de Bioética a estudiantes del IPC de la especialidad de Biología y de la especialidad de Química, lo cual corresponde a la fase 2 del Modelo de Desarrollo Instruccional. Aunque la idea a futuro es implementar la Bioética en todas las especialidades, las investigadoras comenzaron por estas especialidades, ya que son los alumnos que también toman el curso de CTS. En Alvarado et al. (2017) se describe el diseño del curso, cuyo objetivo es despertar la creatividad de los estudian- 
tes y motivar el desarrollo de capacidades propias a su formación en cuanto a actitudes de tolerancia y comprensión (Chaparro, 2011).

En cuanto a estrategias pedagógicas, el curso sigue la tendencia del enfoque principialista por considerar que son aplicables a la sociedad (Sola y Camilo, 2015), en especial en los análisis acerca del impacto de los avances científicos y tecnológicos (Molina, 2011). El enfoque incluye los cuatro principios de Beauchamp y Childress (Royes y Qui, 2019) los cuales son: autonomía, beneficencia, no maleficencia y justicia (Alvarado et al., 2017); además, se incluyen dos que se encuentran en el Código de Ética para la Vida de la República Bolivariana de Venezuela (2011), que son responsabilidad y precaución. Lo principios son utilizados para promover el respeto por la vida cotidiana y las diferencias humanas, con lo cual los estudiantes pueden involucrarse en el cuidado de la vida (Cerchiaro, 2011; García, 2012).

El siguiente punto que se tomó en cuenta para el diseño del curso estaba relacionado con la metodología y las estrategias didácticas que se podían incluir; es decir, cómo enseñar bioética; esto incluye definir el rol del docente, el cual debe distinguirse por incentivar el debate y respetar las distintas opiniones, ya que no hay una sola solución para los casos. Por eso es tan importante el abordaje transdisciplinar de la situación para reflexionar acerca de los valores involucrados. Solo así puede esperarse que el conocimiento científico pueda modificarse a conocimiento ético (Orozco, 2016).

En cuanto a las estrategias, se plantean distintos métodos para la enseñanza de la bioética, los cuales incluyen: debates, clases magistrales, seminarios, estudio de casos, presentaciones en grupo, debates de textos y de películas, simposios y conferencias (Caramico, Zaher y Rosito, 2007).

Con relación a la metodología, se incluyen las siguientes: Modelo por competencias
(Gómez y Royo, 2015); Aprendizaje de la Bioética Basado en Problemas (ABBP) (Corral, Brito y Franco, 2016); Enfoque casuístico (Salort y Maluf, 2014); Enfoque humanista-integral, que considera importante conocer las bases antropológicas y las filosóficas que permitirán tomar decisiones sobre una conducta buena o mala. Esto incluye el conocer la importancia de las leyes (aspecto jurídico que no siempre va de la mano con la ética) y de las religiones para una visión integral del caso (Salort y Maluf, 2014; Gracia, 2019). El énfasis principal para una correcta participación en los temas de bioética no radica tanto en las metodologías, sino que descansa en el proceso y en el rol del profesor como guía y compañero de aprendizaje (laeducacionpintabien, 2018).

En el diseño del curso propuesto en esta investigación, la metodología de análisis de casos se centra en el enfoque humanistaintegral; sin embargo, se consideraron aspectos de los diferentes enfoques, ya que el modelo considera en primer lugar, la determinación del contexto donde se da la situación. En segundo lugar, se plantea el dilema. En tercer lugar, se analizan los principios involucrados (valoración ética), las leyes que están relacionadas con el caso (aspecto jurídico) y la propia postura del que analiza (valores personales). La integración de los tres análisis es lo que lleva a la solución del dilema que no siempre será la mejor; en algunos casos será la de menores consecuencias (Alvarado et al., 2017).

En cuanto a la pedagogía aplicada como método, primero se tomó en cuenta la importancia de la comunicación para el proceso de enseñanza y aprendizaje; para muchos autores el proceso comunicativo es pluridisciplinar y transdisciplinar (Ribot, Pérez, Rousseaux y Vega, 2014). Uno de los representantes de los estudios en comunicación es Mario Kaplún, con influencia del constructivismo de Vygotsky, Bruner y Ausubel y de la pedagogía crítica de Freire; para Kaplún, el educador se convierte en el acompañante y guía para suministrar herramientas que le permiten al 
educando construir sus propias estructuras de significado (EL NACIONAL, 2016), por lo que puede tomar sus propias decisiones y así "aprende a aprender" (laeducacionpintabien, 2018).

El proceso de comunicación en la Bioética debe partir de la pedagogía crítica, la cual busca el desarrollo de las habilidades críticas y reflexivas de los estudiantes aportando conocimientos y cultura que son significativos para el educando (Significados, 2018; Editorial Definición MX, 2016). Los representantes de esta corriente son: Peter Mc Laren (canadiense), Henry Giroux (estadounidense) y Paulo Freire (brasilero) (Significados, 2018). Mc Laren señala que el conocimiento a impartir debe ser importante, pertinente y crítico para poder promover la transformación (Torres, 2019). Por su parte, Giroux señala que la educación debe ser comprometida, crítica y revolucionaria para que permita el desarrollo crítico, así los educandos podrán ser ciudadanos preocupados por afrontar asuntos sociales y estar dispuestos a extender el significado; además, señala el rol del docente como guía en el proceso (Giroux, 2017; Torres, 2019; Bandala, s.f.).

El curso objeto de estudio en esta investigación fue diseñado con estrategias enmarcadas en la reflexión crítica como parte de la adquisición de competencias genéricas en la educación superior. Para esto, se consideró el uso de incidentes críticos que son casos con ciertos significados que van a crear un conflicto con el pensamiento o comportamiento de los alumnos (en el curso de bioética se manejan casos reales). El incidente lleva a un dilema desorientador que facilita el debate guiado por el docente, produciéndose una respuesta consensuada en la que todos los participantes argumentan sus opiniones $\mathrm{y}$, al final, pueden decantarse por la que se considera la mejor respuesta (Deeley, 2016).

Los pasos para la reflexión crítica durante el debate en el aula incluyen: a Describir el acontecimiento o el caso.

Hacer una observación objetiva de lo descrito considerando las emociones que acompañan tales acontecimientos (lo que pone en juego creencias, valores, ideas y hasta comportamientos).

Considerar repuestas alternas y potenciales hasta encontrar la respuesta apropiada, lo que muchas veces cambiará las estructuras previas. Implica reevaluación del acontecimiento original (Deeley, 2016).

En la práctica docente, la reflexión crítica gira en torno a un contenido, los problemas, las respuestas existentes, las respuestas potenciales y hasta las posibles alternativas, porque lo que se busca es que los estudiantes sean pensadores críticos (Deeley, 2016).

Para el curso, se incluyeron dos estrategias importantes de la pedagogía crítica: el análisis de casos y la reflexión. El análisis de casos bioéticos es una estrategia implementada por Schmidt (2008) como una forma de sistematizar la información, el análisis y la comparación de distintos puntos de vista priorizando la vida y la protección al más vulnerable; asimismo, señala que el análisis de casos tiene como fundamento el enfoque fenomenológico al que se suma la comprensión hermenéutica de la realidad. El mismo autor propone un modelo centrado en el proceso de la decisión moral que es la que permite que la persona se apropie del conocimiento moral a través de una serie de operaciones intencionales. A partir del modelo planteado, Alvarado, Partida y Ochoa de Toledo diseñan una adaptación del mismo con fines estrictamente pedagógicos, pero manteniendo el objetivo del método (Alvarado et al., 2017).

En cuanto a la reflexión, podría considerarse una escritura reflexiva. No es exactamente un diario, pero si es reflexiva por cuanto en ella el alumno se autoevalúa, 
con lo cual es consciente de lo que aprende. Como guía, se dan a los estudiantes los puntos que deben incluir; pero, se da flexibilidad en cuanto a su presentación, redacción, longitud y en cuanto a otros aspectos que deseen incluir. La autoevaluación queda evidenciada al señalar los conocimientos adquiridos y los cambios de concepción, lo que representa un elemento importante para considerar el aprendizaje crítico (Deeley, 2016; Giroux, 2017; Torres, 2019). Dentro de las reflexiones se incluye la reflexión final del curso, la cual representa un escrito libre acerca de las impresiones del curso culminado, el aprendizaje logrado y cualquier otro aspecto de interés.

Tanto los debates y el análisis de casos como las reflexiones cumplen con los objetivos del aprendizaje crítico por cuanto implican la reflexión crítica y demuestran la existencia del pensamiento crítico que vincula lo conceptual teórico con la práctica cotidiana y sus experiencias (Deeley, 2016; Giroux, 2017; Torres, 2019).

El curso fue propuesto y aceptado como Optativo de Profundización para los estudiantes de Biología, administrado por la Cátedra de Biología Celular con el Código BID205 en el 2011. Posteriormente, para el 2012, se aceptó el curso para los futuros docentes de Química con el código EQUI275.

Siguiendo el método de Müller de González y Szczurek (1989), citado en Castro y Guzmán de Castro (2001), esta investigación presenta los pasos 3 y 4 , es decir, la validación y evaluación de la aplicación de la propuesta, en este caso, del curso. Primero, se indican las características y resultados de la aplicación piloto del curso. Luego, a partir de los resultados obtenidos, la implementación del curso; finalmente, se presentan los resultados del mismo a la luz de las percepciones de los estudiantes.

Ya que hemos descrito brevemente en qué consistió la implementación del curso y las estrategias aplicadas para el logro del involucramiento y compromiso de los participantes, el objetivo general de este presente estudio es analizar las percepciones de los alumnos con relación al curso de bioética para estudiantes de pregrado de la especialidad de Biología y de Química del Instituto Pedagógico de Caracas de la Universidad Pedagógica Experimental Libertador. Como objetivos específicos se propone: relatar la experiencia del curso piloto con el fin de mostrar los ajustes que se hicieron para implantar la asignatura de Bioética; sintetizar los resultados de la implementación del curso de Bioética y ofrecer recomendaciones sobre las clases y metodologías utilizadas a la luz de los objetivos del curso y de las percepciones de los estudiantes. En ese mismo orden, primero se verá el modelo del método utilizado; más adelante, presentamos los resultados cualitativos de la percepción, así como el análisis de sus opiniones. Finalmente, presentamos las conclusiones a las que arribamos, así como unas recomendaciones como estímulo para que la universidad se proponga implementar esta asignatura en la formación de futuros docentes.

\section{Etapa 3 del Modelo del Diseño Instruccional}

\subsection{Método}

La investigación es cuasi experimental cualitativa con estudio de campo y se enmarca en el paradigma interpretativo porque se pretende recabar la información de los estudiantes para su posterior análisis e interpretación de la realidad del curso de bioética. Se trata de un estudio de tipo cuasi experimental, aplicado a secciones intactas del curso de Bioética del Instituto Pedagógico de Caracas.

Para el procesamiento de los datos y su análisis se manejó el Método de Comparación Continua (MCC propuesto por Glaser y Strauss (1967) y Straus y Corbin (1998). 
La población seleccionada para las etapas 3 y 4 fueron todos los estudiantes inscritos en el curso de Bioética desde 2009 hasta 2016.

Para efectos de validación de la propuesta correspondiente a la fase 3 del modelo de Müller de González y Szczurek de 1989 (citado por Castro y Guzmán de Castro, 2001), el curso se aplicó como prueba piloto en el semestre 2009-I. Solo tuvo cinco participantes de biología (total de la población del curso de Bioética), por lo que el total de la población se utilizó para recabar información que permitiera hacer ajustes a la propuesta para futuras aplicaciones. La prueba piloto incluyó las siguientes estrategias: exposiciones, debates de películas, análisis de casos y reflexiones críticas.

Para la fase 4 de implementación de la propuesta, se tomaron en cuenta los resultados de la prueba piloto para hacer ajustes a la misma. La aplicación del curso considerada para esta investigación va desde el semestre 2011-I hasta el 2016-I (total de estudiantes: 113); en todos los semestres se utilizaron las mismas estrategias de la prueba piloto, pero se agregó conferencia de expertos. Igualmente, en los primeros semestres de aplicación del curso (2011-I hasta 2012-I), se aplicó una encuesta acerca de las expectativas del curso. El material recabado se organizó por semestre y por actividad para mayor facilidad del procesamiento de los datos.

\subsection{Resultados}

La investigación culmina con la reflexión acerca de lo hallazgos obtenidos. El primer paso, luego de la organización de los documentos, fue tabular la información; a partir de esta se procedió a categorizar según lo planteado por Strauss y Corbin (1998). La información posteriormente se estudió y se hizo el análisis correspondiente apoyando los hallazgos obtenidos en las referencias teóricas.

Resultados de la fase 3. Aplicación piloto del curso para validación de la propuesta del mismo.
Durante el curso, las estrategias de evaluación incluyeron: análisis de casos, exposiciones por parte de los estudiantes, reflexión de cada uno de los temas abordados y una reflexión final del curso. La evaluación fue formativa en cuanto al hecho de que el estudiante tenía oportunidad de mejorar reflexiones y análisis de casos; y, sumativa, en el caso de las exposiciones. Finalmente, se les pidió la reflexión final acerca del curso.

Los aspectos cualitativos resultantes de las distintas estrategias incluyen:

1 En las reflexiones de cada tema los estudiantes colocan mucha información sobre lo que aprendieron, lo que facilita la autoevaluación por parte del estudiante y la evaluación por parte del docente. Igualmente, en estas reflexiones los estudiantes señalan la necesidad de plantear los aspectos legales y la importancia de respetar la diversidad de opiniones. Se incluyen cambios de concepción en los temas específicos.

Las distintas exposiciones realizadas por los mismos estudiantes permitieron ampliar la visión acerca del tópico tratado ya que creían que la bioética era solo para el área salud. Consideraron que en el caso de las exposiciones de los estudiantes se requería más tiempo y más profundidad para abordar diversos aspectos. Recomendaron el uso de videos para hacerlas más dinámicas.

Los estudiantes elaboraron los análisis de casos; incluso, una alumna logró establecer su propio modelo. Sin embargo, señalaban la maleficencia como principio bioético, lo que indica la confusión entre acto y principio bioético. Y en el caso de la opinión bioética, en varias ocasiones, la misma fue muy escueta aparte de que no se manejaron los argumentos que hicieron en las valoraciones. 
4 En la reflexión final, consideraron que Bioética es la mejor materia de integración y análisis del conocimiento y que debería implementarse como un código de conducta humana en todas las especialidades. Señalaron la importancia del curso para aprender a respetar la diversidad de opiniones.

Finalmente, en cuanto al contenido, dos estudiantes señalan que creían que era otra materia para completar créditos, pero, en realidad, es un curso que ofrece herramientas para análisis de dilemas y para el trato de recursos humanos que debemos manejar los docentes. El número pequeño de estudiantes inscritos, parece evidenciar que los alumnos creían que era otra materia del área pedagógica como filosofía, por lo que no la escogen. De hecho, en la medida que se sigue ofreciendo el curso, el número de inscritos aumenta por los comentarios de los estudiantes que la habían cursado. Al respecto, para confirmar los prejuicios de los alumnos, se determinó la necesidad de realizar una encuesta a los educandos para conocer acerca de las expectativas que tenían del curso al comenzar.

Para los efectos del curso como prueba piloto, los resultados fueron muy satisfactorios, a pesar de que la muestra era muy pequeña, ya que se lograron los objetivos planteados; además el cumplimiento de asignaciones y el cómo fueron entendidas se vio reflejado en las calificaciones obtenidas; sin embargo, se notaban dificultades para diferenciar la valoración personal, los aspectos legales y los principios bioéticos con los que debía argumentarse la opinión bioética. En el análisis del dilema no aparecían los principios; los colocaban en la posible solución del dilema sin discriminar cuáles eran los que correspondían.
La prueba piloto del curso permitió evaluar el uso de las estrategias planificadas, el desenvolvimiento y responsabilidad de los estudiantes; además, ofreció la oportunidad de ver los aspectos que podrían ser mejorados en la implementación definitiva del curso tales como la forma de abordaje a los dilemas éticos y el establecimiento de pautas para las exposiciones de los estudiantes.

Resultados de la Etapa 4 del Modelo del diseño instruccional: Implementación de la propuesta.

Los semestres en los que se dictó el curso incluyeron desde el 2011-1 hasta el 2016-I y el grupo de estudiantes estuvo constituido por toda la población de futuros docentes de Biología y de Química, cursantes de Bioética, ya que desde el 2012 se aprueba Bioética como curso optativo de profundización para los estudiantes de Química. Para efectos de la evaluación, se consideraron parámetros que debían ser incluidos para cada actividad: así, las exposiciones debían incluir tanto el aspecto conceptual como el bioético, vinculado a una problemática; los casos debían seguir las pautas del modelo pedagógico para análisis de casos bioéticos, según Alvarado et al. (2017); las reflexiones debían incluir aspectos tales como qué aprendí; cambios de concepción; qué me gustó; recomendaciones para presentar el tema. En función de los aspectos considerados, se asignaba el puntaje a la actividad, para cumplir con la evaluación como requisito académico.

\subsection{Recolección y análisis de las percepciones}

El análisis se hizo acorde a los elementos considerados para la evaluación del curso y la encuesta inicial.

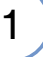

Encuesta para cursantes de Bioética 2011-I, 2011-II y 2012 II $(n=51)$ con preguntas acerca de las expectativas del curso, lo que les había parecido 
hasta ese momento (llevaban tres semanas del curso), si lo consideraban una asignatura importante $\mathrm{o}$ necesaria y si lo recomendarían.

Los resultados fueron los siguientes:

\section{Pregunta: ¿Qué expectativas tenías en relación a este curso?}

Resumiendo lo que expresan los estudiantes, surgen los siguientes puntos: en el primer semestre de aplicación de la encuesta, creían que el curso era de doctrinas de filosofía, que solo serviría para completar créditos; en semestres posteriores, señalan que podría ser una asignatura que los ayudaría en su rol de docentes, en las relaciones de la biología con la ética, en su vida y entorno, en la investigación; algunos pensaron que era divertido, o que presentaba muchos aspectos científicos, importantes para los investigadores; otros pensaron que era un curso para aprender sobre el marco legal y su aplicación en los casos bioéticos.

En conclusión, las expectativas eran diversas; en el primer semestre de aplicación de la encuesta había un total desconocimiento acerca del contenido y utilidad del mismo. Pero, a partir del segundo semestre de aplicación de la encuesta, aparecen expectativas del curso en cuanto a los objetivos del curso, casos bioéticos, marco legal, y a la ética presente en todos los aspectos de la vida. Estos señalamientos incluían los comentarios y experiencias de compañeros que ya habían tomado la asignatura.

\section{Pregunta: ¿Qué te ha parecido el curso?}

Como los alumnos ya tenían tres semanas en el curso, se hizo la segunda pregunta, de la cual surgieron las siguientes consideraciones: el curso les resulta más interesante e importante de lo que se plantea en las expectativas. Algunos estudiantes hablaron de clases un poco tediosas, lo que posiblemente se deba a las clases de fundamentación del curso que resultan ser menos participativas. Otros plantean que es una asignatura importante para el desarrollo y crecimiento personal; también plantean que hace pensar en casos del entorno, que cambia la visión acerca de los científicos y sus investigaciones, que se presta al debate y la discusión, que enseña a hacer análisis bioéticos con la inclusión de aspectos legales.

Pregunta: ¿Crees que la información suministrada en las primeras clases es relevante? ¿Por qué?

Los aspectos considerados en las respuestas incluyen afirmaciones de que sí es importante, ya que las primeras clases dan el sustento teórico para argumentar una posición ante los casos bioéticos que se presenten; sin ellos, los argumentos serían solo desde la óptica personal por lo que resultarían muy subjetivos; además, enseña la importancia del marco legal.

\section{Pregunta: ¿Crees que bioética debería ser un curso optativo u obligatorio? ¿Por qué?}

El resumen de las respuestas incluye: todos los estudiantes respondieron que debía ser un curso obligatorio no solo para Biología y para Química, sino también para todas las especialidades, toda carrera universitaria y para todo público, porque enseña aspectos legales que todos deberíamos conocer; es un curso que ayuda a crear conciencia con lo que se puede mejorar nuestra sociedad, ayudar al respeto a los demás por lo que algunos cambiarían Ética y Docencia por Bioética.

\section{Pregunta: ¿Recomendarías un curso o taller de Bioética? ¿Por qué?}

La gran mayoría de los encuestados respondieron que sí lo recomendaría. Esta respuesta consideró la opción taller, ya que sería una forma de propagar esta disciplina en la comunidad en general y así resaltar la importancia y la necesidad de la ética en la vida. Algunos sugieren temas de la actualidad nacional tanto como de la internacional. 
Este instrumento solo se aplicó en los semestres iniciales, ya que, por lo novedoso, había muchas expectativas e información errada acerca del curso. Parecía interesante recoger esas impresiones y de esa forma comparar lo que pensaban al final de la asignatura, a través de la reflexión final. La encuesta no se aplicó en los siguientes semestres; sin embargo, de forma oral se hacía como introducción al curso; los resultados que se recogieron eran iguales a los descritos en cuanto a las expectativas e importancia. En la misma medida que se aplicaba el curso, disminuía la probabilidad de señalamientos de expectativas erróneas; incluso, se notó un incremento de la matrícula. El incremento luego deja de observarse, pero es debido a otra razón: los alumnos no terminan la carrera porque se incorporan al medio laboral o emigran buscando mejores condiciones económicas que las que ofrece Venezuela en los últimos años.

2 Estrategias manejadas en clase. Curso de Bioética: semestres 20112016.

La información acerca de las estrategias fue recogida a través de la observación participante en las clases y a través de las asignaciones que el docente evaluaba (Análisis de caso, reflexiones, exposiciones).

\section{- Análisis de caso}

Una de las mayores inquietudes que surge en las primeras aplicaciones del curso fue la realización de los análisis de caso. Así se observa que, al igual que en la aplicación piloto de la asignatura, en los semestres del 2011 y del 2012 a veces no aparecían los principios en el análisis, sino que aparecían en la opinión bioética; en muchas oportunidades no se planteaba el dilema a resolver. Algunos, hablan de la maleficencia como un principio bioético que no lo es. Hubo casos de opinión bioética muy escueta y solo desde el punto de vista personal, sin argumentos legales o sin los principios bioéticos; estos aparecían en el análisis, pero no como argumentos en la opinión bioética.

Esto llevó a Alvarado et al. a diseñar un modelo pedagógico para el análisis de los dilemas bioéticos (2017). A partir del uso de dicho modelo en el semestre 2012 II, se empezaron a corregir los problemas de los análisis bioéticos, dándole mayor peso a la opinión; esta última representa la posible solución al dilema, por lo que deben aparecer los argumentos legales y los principios bioéticos afectados por la decisión. El modelo se apoya en el enfoque humanista-integral ya que se maneja tanto la valoración filosófica representada por los principios como el marco jurídico acorde al contexto del caso presentado; igualmente, se consideran los aspectos religiosos como parte de la valoración personal (Salort y Maluf, 2014). El otro aspecto siempre considerado fue tratar casos reales a partir de casos clínicos, casos de las diversas formas de vida y casos ambientales para una mayor integración de la bioética a la vida cotidiana.

El esquema contribuyó a la mejora de los análisis y por eso se utilizó primero como ensayo de una propuesta de modelo y, posteriormente, como modelo pedagógico para el curso.

\section{- Otras estrategias}

El resultado de las distintas estrategias resultó muy satisfactorio, tal como lo manifiestan los mismos estudiantes. Así, expresaron que fue excelente el utilizar diversos expertos en el curso; les gustó la diversidad de temas; en alguna oportunidad, se llevó a los estudiantes a una expotatoo (evento de tatuadores), para estar más en contacto con la realidad y así poder analizar casos relacionados con el tópico; en este sentido, los estudiantes manifestaron total satisfacción con la actividad. El análisis bioético de películas les dio otra forma de ver situaciones reales y ficticias, en las que claramente la Bioética está involucrada, por lo que la consideraron una estrategia muy 
útil. Muchos comentaron el uso del blog que se abrió para los estudiantes de Bioética donde podían aportar sus ideas y opiniones libremente. Les pareció que en todos los temas se podían expresar distintas visiones. En resumen, las estrategias manejadas por las docentes, según los estudiantes, representan una forma de sentirse protagonistas en la clase generando el aprendizaje a través de una mejor interacción grupal, y apoyan lo planteado por diversos autores como Orozco (2016) y Caramico et al. (2007).

Estas percepciones fueron obtenidas de las reflexiones finales del curso.

\section{(3) Aspectos señalados en las reflexiones.}

Las reflexiones incluyen tanto las que hacían por tema como la reflexión final. Las primeras eran estructuradas en preguntas: ¿Qué aprendí? ¿Qué me gustó? ¿Qué no me gustó? ¿Cuáles fueron los cambios de concepción? ¿Qué recomendaciones haría para este tema? En cuanto a la reflexión final, era totalmente abierta y allí podían hablar del curso, de las estrategias, de las docentes y de los expositores; de allí se extrajo una variedad de información, y la más importante es que los estudiantes consideran que deben existir reflexiones en todas las asignaturas para poder expresarse y autoevaluarse. Todo el material fue tabulado y organizado en categorías que incluyen: su percepción con relación al curso, aprendizaje adquirido, bioética como disciplina, rol del docente y recomendaciones para la asignatura.

\section{- Con relación al curso de Bioética}

Algunos aspectos importantes señalados incluyen que el curso hace posible que se establezca una conexión entre el mundo real y el mundo académico; además, es integrativo de diversas disciplinas, motiva el debate y el análisis, nos ayuda a cambiar sensibilizándonos con todos los aspectos que conforman nuestro día a día; es fácil de entender y llevar ya que se manejan casos reales que creemos lejos de nuestro entorno; aborda preguntas que alguna vez se han hecho, pero desde la óptica de la ética haciendo posible la madurez del pensamiento crítico, que facilita la transferencia a todos los ámbitos, incluyendo el educativo. Permite la expresión de las diversas opiniones, a ponerse en el lugar de los demás y aprender a partir de sus vivencias. En este sentido, el curso modifica conductas personales, conceptos, puntos de vista, paradigmas; y, además, estimula el obtener una buena nota y no solo obtener la mínima aprobatoria.

Los estudiantes señalan que el curso debería ser obligatorio para todas las especialidades por la actual crisis de valores; el ser humano se ha enfocado tanto en avanzar tecnológicamente y en otros aspectos, que se ha olvidado de los valores éticos para lograr una mejor sociedad. El curso aporta las herramientas necesarias día a día para la toma de decisiones con argumentos sólidos.

El curso permite crear conciencia social de la acción transformadora de la educación y el rol protagónico y determinante que desempeña el docente, lo que apoya la pedagogía crítica según Deeley (2016), Giroux (2017) y Torres (2019).

En resumen, la aceptación del curso fue total. Ninguno expresó que no le gustara. En líneas generales puede decirse que la asignatura les deja huellas para cambios personales y académicos, lo que parece evidenciar que la utilización de la pedagogía crítica puede lograr una educación transformadora en los estudiantes.

\section{- Aprendizaje adquirido en el curso}

La mayoría de los estudiantes expresa en las reflexiones algunos comentarios puntuales que incluyen el aprendizaje acerca de los valores y cómo ellos influyen en las decisiones humanas; el poder comparar los primeros análisis bioéticos con los últimos, les permite ver su crecimiento en cuanto a la realización de los mismos y la importancia 
de considerar los principios bioéticos y el marco legal; señalan también el conocimiento acerca de los comités bioéticos y el rol que cumplen en las instituciones presentes. Además, el abordaje de diversos temas a través de casos reales, los llevan a entender que no hay dos extremos ni dos tonos, sino que hay matices, lo que los ayuda para su rol docente y su crecimiento personal; un punto fundamental del aprendizaje en el curso es la realización de los análisis bioéticos.

Los estudiantes en general sienten que el aprendizaje va más allá de la información. Es un curso de formación, ya que la mayoría señala los múltiples cambios que le genera tanto en el ámbito personal como en el académico, lo cual va acorde a lo que se plantea en la pedagogía crítica: motivación a los estudiantes para cuestionar, criticar y asumir posturas con argumentos sólidos tal como se ha señalado en distintos apartados de este trabajo.

\section{- Bioética como disciplina}

En las reflexiones finales, los estudiantes hicieron mucho énfasis en la bioética como disciplina. Así, por ejemplo, la definen como el estudio de todas esas problemáticas características de la especie humana; como un espacio para la discusión crítica de las mismas, lo que permite desarrollar nuevas estrategias para el abordaje de casos en el ámbito pedagógico. La bioética ayuda a educar más allá de contenidos procedimentales y teóricos; en este sentido, apoya a la educación desde una perspectiva holística de la enseñanza, en especial si se trata de áreas científicas.

Finalmente, algunos señalan que si hubiese más cursos de bioética los tomarían todos por la experiencia ganada. Además, que debemos ser los propagadores de bioética en nuestros salones de clase y círculo social.

\section{- Docente}

Uno de los aspectos más resaltantes en las reflexiones de los estudiantes es el rol fundamental del docente para este tipo de curso. Ellos señalan que el docente no solo debe dominar el aspecto teórico del curso, sino que también debe tener la habilidad para el uso de herramientas y recursos, además de ser abierto y comunicativo (estas características la señalan a partir de la experiencia con las docentes del curso). Es el docente el que puede guiar para el cambio, para que puedan sensibilizarse con las situaciones y con esa mejora que como humanos debemos alcanzar, tal como lo plantea Kaplún (EL NACIONAL, 2016).

\section{- Recomendaciones para el curso}

A partir de las reflexiones, se hizo un breve resumen de las recomendaciones que hacen los estudiantes para el curso. Puede notarse que, aunque dan sugerencias de nuevas estrategias, en líneas generales, los educandos aspiran a una bioética más allá de la asignatura.

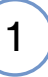

Los temas presentados deberían ser parte de la carrera para hacer a los docentes más tolerantes, para evitar colocar etiquetas que conllevan a la discriminación y para prepararlos para muchos casos que se presentan en su ámbito de trabajo. Más aún debería ser para todo público y, en el caso del ámbito educativo, debería comenzar en el nivel básico como parte del proceso de formación del ciudadano para hacerlo más humanista respecto al planeta y a la convivencia con todos los seres humanos.

2 Se podrían proponer áreas de investigación en bioética y proyectos en el área para seguir el trabajo de esta disciplina y afirmar el inmenso aprendizaje que deja en los estudiantes.

Podría hacerse el cierre del curso con un caso libre propuesto por cada estudiante (real o ficticio). Podrían 
incluirse mesas de trabajo y dramatizaciones.

\section{Conclusiones}

La Bioética como curso puede contribuir a manejar la parte humanista junto a los avances científicos y tecnológicos, formando a los profesionales como ciudadanos capaces de tomar decisiones argumentadas que busquen causar el menor daño posible. Siempre y cuando se maneje la pedagogía crítica, este tipo de curso permite formar a las personas haciéndolas más tolerantes, respetuosas de las ideas diferentes, a través de un aprendizaje basado en la discusión y el debate. Es decir, la asignatura incluye contenidos actitudinales al involucrar a los estudiantes en las situaciones y reflexionar sobre sus posibles soluciones, contenidos dirigidos a la formación más que a la información, en concordancia con lo planteado por Deeley (2016), Giroux (2017) y Torres (2019).

2 Las percepciones de los estudiantes permiten confirmar que el uso de la pedagogía crítica es fundamental para este tipo de curso, ya que pueden concientizar que la visión personal no es la que conduce a la mejor respuesta; por tanto, hay un conflicto entre la respuesta y lo que se creía. Esto podría llevar a ser más abierto a distintas opciones, al respeto de otras opiniones y al cambio hacia un profesional más comprometido con su entorno social (Deeley, 2016, Giroux, 2017 y Torres, 2019).

3 A través de las reflexiones, los estudiantes señalan los cambios logrados, lo que sugiere un aprendizaje crítico. De hecho, trasladan lo aprendido a situaciones vividas en su día a día y hasta comparten con amigos y familia los tópicos de la asignatura, según lo que señalan algunos estudiantes. Sin embargo, no hay garantía de que con la reflexión crítica el estudiante cambie; y si cambia, tampoco se puede determinar hasta qué punto se da ese cambio; aun así, no hay duda de que esta estrategia si puede llevar al cambio (Deeley, 2016).

\section{Recomendaciones finales}

Se puede evidenciar que el curso promueve cambios en los estudiantes. Sin embargo, el alcance de este estudio no permite conocer si esos cambios perduran a largo plazo en ellos, ya que se desconoce si en otras asignaturas se plantea el aprendizaje crítico. Con solo un curso pueden promoverse cambios, pero pueden diluirse si no hay refuerzos en otros cursos donde pueda involucrarse la realidad como parte del aprendizaje y los aspectos éticos involucrados. Sin embargo, el hecho de que se generan productos posteriores al curso, nos conduce a pensar que este va más allá de un aprendizaje para un semestre. De allí que sugerimos talleres para docentes de tal forma que todos puedan aplicar estrategias que consoliden los cambios promovidos por la bioética.

La aplicación del curso ha resultado muy satisfactoria para nosotras las docentes, porque, aunque pensamos que a la bioética todavía le falta mucho por hacer, por lo menos se ha empezado a considerar la importancia de la disciplina para la formación de nuestros docentes y nuestros investigadores. Por ahora hay pocos productos, pero se espera que en el futuro se sigan sumando nuevos aportes, ya que se cuenta con seminarios de Bioética en la Maestría de Enseñanza de la Biología, se abrió un curso de Bioética en Educación Integral y se están incluyendo los aspectos bioéticos de la investigación como exigencia de acuerdo al nuevo reglamento de investigación. La máxima aspiración es que bioética sea un curso obligatorio para todas las especialidades de la universidad y que poco a poco pueda transversalizarse esta 
disciplina a través del nuevo currículo universitario.

\section{Referencias}

Alvarado, G., Partidas, G. y Ochoa M. (2017) Una metodología para discutir dilemas éticos. Revista de Educación Superior y Sociedad. 17: 116-133.

Bandala F., O. (s.f.). Las prácticas pedagógicas en el aula: ¿Un paso a la libertad o a la dominación? Monografías.com.

Bermúdez de Caicedo, C. (2006). Necesidad de la bioética en la educación superior. Acta Bioethica 12 (1): 35- 40.

Caramico, H.J.; Zaher, V.L. y Rosito, M.M.B. (2007). Ensino da Bioética nas faculdades de medicina do Brasil. Bioethikos, 1(1), 76-90.

Casado González, M. (2011). Bioética y Educación. Sobre la necesidad de adoptar una concepción de la bioética flexible y que promueva la educación en los principios de la Declaración Universal sobre Bioética y Derechos Humanos. Revista Latinoamericana de Bioética. 11 (2): 62-71.

Castro, S. y Guzmán de Castro, B. (2001). ¿Es la Tecnología Útil en la Educación? Revista de Investigación No. 49: 11-37

Cerchiaro, D.M. (2011). Elementos pedagógicos y didácticos para la inclusión de la bioética en el currículo del programa de enfermería de la Universidad Popular del Cesar. Tesis de Maestría. Disponible en https://docplayer.es/92649846-Elementos-p edagogicos-y-didacticos-para-la-inclusion- $d$ e-la-bioetica-en-el-curriculo-de-enfermeriade-la-universidad-popular-del-cesar.html

Chaparro, N. (2011). El aprendizaje de valores en: Docencia de la bioética en Latinoamérica. Experiencias y valores compartidos. Santiago de Chile, FELAIBE. Sociedad Chilena de Bioética y Fundación Ciencia y Vida.
Código de Ética para la Vida. (2011). Ministerio del Poder Popular de Ciencia y Tecnología de la República Bolivariana de Venezuela.

Corral, Y; Brito, N. y Franco, A. (2016). Bioética: un concepto que tiende a ser relegado en la educación universitaria venezolana. Universidad de Carabobo ValenciaVenezuela. Jornada Divulgativa de Producción Intelectual de Profesores e Investigadores de la FaCE-UC.

Deeley, S.J. (2016). El Aprendizaje-Servicio en educación superior. Teoría, práctica y perspectiva crítica. Madrid. Narcea, S.A. de Ediciones

Editorial Definición MX. (11/12/2016). Definición de Pedagogía Crítica - Qué es y Concepto Pedagogía Crítica. Sitio: Definición MX. Ciudad de Mexico.

EL NACIONAL. (2016, marzo 6). Pedagogía de la Comunicación. [Publicación en línea]. .

García, J.J. (2012). Bioética Personalista y Bioética Principialista. Perspectivas. Bioetica WeB. [Publicación en línea]. Recuperado de: https://www.bioeticaweb .com/bioactica-personalista-y-bioactica-prin cipialista-perspectivas/ ]

Giroux, H. (2017). Su visión educativa en diez puntos. Aula Planeta. Innovamos para una educación mejor.

Glaser, B y Strauss, A. (1967). The discovery of grounded Theory: strategies for qualitative research. New York: Aldine Publishing Company. Traducción original: Floreal Fomi. Edición, revisión y ampliación: Ma José Llanos Pozi. Universidad de Buenos Aires.

Gómez, V. y Royo, P. (2015). Construyendo espacios de diálogo: una propuesta de enseñanza de la bioética. Acta Bioethica, 21 (1), 9-18.

Gracia, D. (2019). Editorial. EIDON, 52:1-2

Laeducacionpintabien (2018). Libro: Una pedagogía de la comunicación de Mario Kaplún. Ley Orgánica de la Educación (2009). Gaceta №: 5.929 Fecha de Gaceta: 15-ago-09. 
Molina R. N. (2011, diciembre). ¿Qué es la bioética y para qué sirve? Un intento de Pedagogía Callejera. Revista Colombiana de Bioética. 6 (2): 110-117.

Orozco M.B. R. (2016). La bioética en el ámbito escolar. OEI Iberoamérica divulga Plan Socialista de la Nación 2007-2013. República Bolivariana de Venezuela.

Postigo, E. (2015). Bioetica definicion: Que es bioética, Concepto de Bioética y corrientes actuales. Bioètica Web.

Ribot, V. C.; Pérez M., M.; Rousseaux M., E. y Vega G. (2014). Educación Médica Superior. Educ Med Super vol.28 no.2 Ciudad de la Habana.

Royes y Qui, A. (2019). Comentarios al libro "Principios de ética biomédica", de T. Beauchamp y J. Childress. Observatori de Bioètica i Dret. Universitat de Barcelona.

Salort, J. C. y Maluf, F. (2014). De la enseñanza de la bioética a la educación bioética. Reflexiones sobre los desafíos en los posgrados en Bioética. Revista Latinoamericana de Bioética, vol. 14, núm. 2, 2014, pp. 52-65. Universidad Militar Nueva Granada Bogotá, Colombia.

Sánchez-Migallón, S. (2012). Versión de Archivo. Utilitarismo [Publicación en línea].

Schmidt, L. (2008). Colección Biodiké: hacia una Bioética del Siglo XXI. Separata No. 3: Método de Análisis Holístico e Interpretación de Casos Bioéticos. Editorial San Pablo
Sgreccia, E. (2013). Persona Humana y Personalismo. Cuadernos de Bioética, vol. XXIV, núm. 1, enero-abril, 2013, pp. 115-123 Asociación Española de Bioética y Ética Médica Murcia, España.

Significados. Ciencia y salud (2018). Significado de Pedagogía crítica (Qué es, Concepto y Definición). "Pedagogía crítica". En: Significados.com.

Sola, R. y Camilo, J. (2015). Corrientes y tendencias en la Bioética. En Ética y Vida.

Strauss, A. y Corbin, J. (1998). Bases de la investigación cualitativa. Técnicas y procedimientos para desarrollar la teoría fundamentada. Editorial Universidad de Antioquia. Segunda edición (en inglés): Sage Publications, Inc. (United States, London, New Delhi), 1998 Primera edición (en español): Editorial Universidad de Antioquia, diciembre de 2002.

Torres, H., A. (2019). Pedagogía crítica. MILENIO 2020 años. En línea: https://www.milenio.com/opinion/alfonso-tor res-hernandez/apuntes-pedagogicos/pedag ogia-critica

Wilches Flórez, A M. (2011). La Propuesta Bioética de Van Rensselaer Potter, cuatro décadas después. Opción, Año 27, No. 66 (2011): 70 - 84 ISSN 1012-1587. 